\section{Two episodes of listeriosis in pregnancy and newborns: investigation, problems and considerations}

\author{
Selene Marozzi, ${ }^{1}$ Rita Tolli, ${ }^{1}$ Stefano Bilei, ${ }^{1}$ \\ Daniela Ricci, ${ }^{2}$ Claudia Rossi, ${ }^{2}$ \\ Teresa Bossù'
}

${ }^{1}$ Institute for Experimental Veterinary

Medicine of Latium and Tuscany, Rome;

${ }^{2}$ Local Health Unit Rome B, Italy

\section{Abstract}

In January 2013, 3 strains of Listeria monocytogenes isolated from one mother and her newborn and a second infant arrived at the Institute for Experimental Veterinary Medicine of Latium and Tuscany. At the same time the Local Health Unit Rome B sampled at retail a smoked salmon that was suspected to be involved in the transmission of the disease. The sample was tested for the presence of $L$. monocytogenes (UNI EN ISO 11290: 2005 part 1 and 2). Laboratory investigations have shown that all 3 strains isolated from patients belonged to serotype $4 \mathrm{~b}$ of two different clusters (cluster B for the isolates from mother and child and cluster A to that obtained from another baby), so no epidemiological link was demonstrated in the human cases. The tests conducted on smoked salmon have shown the presence of $L$. monocytogenes serotype 1/2a. Therefore, no correlation has been demonstrated between the reported cases of listeriosis and the food analyzed.

\section{Introduzione}

Si definisce episodio tossinfettivo un evento epidemico o sporadico di natura infettiva o tossica causato dal consumo di cibo. L'Autorità competente può ricevere una segnalazione di sospetta tossinfezione alimentare da varie fonti (per es. privati cittadini, operatori del settore alimentare, sistemi di sorveglianza). A seguito di tale comunicazione prende avvio l'investigazione finalizzata ad identificare le fonti e le modalità di trasmissione e, di conseguenza, a circoscrivere l'evento ed eventualmente intervenire con azioni di gestione del rischio. Secondo le indicazioni della World Health Organization (WHO, 2008), una valutazione completa in corso di tossinfezione alimentare dovrebbe normalmente prevedere tre fasi, ovvero: i) indagine epidemiologica; ii) indagini ambientali e sugli alimenti; iii) analisi di laboratorio.
L'indagine epidemiologica si configura come il momento grazie al quale è possibile sviluppare un'ipotesi descrittiva della storia dell'episodio tossinfettivo. Parallelamente alla stessa, vengono condotte investigazioni ambientali e sugli alimenti con l'obiettivo di definire luogo e cause della contaminazione dell'alimento ed eventuali azioni correttive.

Nel 2012 sono state notificate nell'Unione Europea (EU) 5363 epidemie a trasmissione alimentare che hanno coinvolto 55.453 persone. Di queste un totale di 1642 (3\%) affette da Listeria monocytogenes, con un aumento del $10.5 \%$ rispetto al 2011 (EFSA, 2014). Secondo alcune stime il $99 \%$ dei casi di listeriosi è attribuibile al consumo di alimenti contaminati (Mead et al., 1999). Le malattie da L. monocytogenes nelle donne gravide sono 18 volte più frequenti $(12 / 100.000)$ che nel resto della popolazione (0,7/100.000). Inoltre, il $16-27 \%$ di tutti gli episodi di malattia associati a L. monocytogenes si verificano nelle donne in gravidanza. Casi sporadici ed epidemici di listeriosi nelle donne nel periodo perinatale sono stati riportati con una prevalenza che varia tra l'8,6 e il 17,4/100.000 nati vivi (Ronald et al., 2011). La trasmissione diretta è possibile ma rara. Una donna incinta può trasmettere l'infezione al feto in utero per via trans placentare 0 durante il passaggio nel canale del parto (ANSES, 2011).

Gli alimenti a maggior rischio come quelli, che per loro natura, rappresentano un terreno favorevole alla crescita di $L$. monocytogenes, sono ready-to-eat (RTE) che richiedono refrigerazione ed hanno una shelf-life prolungata, quali ad esempio, prodotti ittici crudi e e affumicati, formaggi a pasta molle, latte non pastorizzato e vegetali crudi (Alleberger e Wagner, 2010).

Nel presente lavoro vengono illustratati esiti, modalità e criticità di un'indagine ambientale, alimentare e di laboratorio successiva all'isolamento di alcuni ceppi di $L$. monocytogenes in pazienti ricoverati presso due presidi ospedalieri romani.

\section{Materiali e Metodi}

Nel gennaio 2013 sono stati inviati da una struttura sanitaria pubblica di Roma al Centro di Riferimento Regionale per gli Enterobatteri Patogeni (CREP) dell' Istituto Zooprofilattico Sperimentale del Lazio e della Toscana, M. Aleandri (IZSLT), 3 ceppi batterici di $L$. monocytogenes isolati da altrettanti pazienti. L'isolamento era stato ottenuto dal sangue di due neonati e dai lochi vaginali di una delle madri. La diagnosi di listeriosi era stata emessa per la madre e per il figlio rispettivamente in data 14/01/2014 e 08/01/2014, mentre per il secondo neonato il 24/12/2013. L'isolamento in
Correspondence: Selene Marozzi, Institute for Experimental Veterinary Medicine of Latium and Tuscany M. Aleandri, via Appia Nuova 1411, 00178 Rome, Italy.

Tel/Fax: +39.06.79099429.

E-mail: selene.marozzi@izslt.it

Key words: Listeria monocytogenes; Listeriosis; PFGE; Investigation.

Received for publication: 10 July 2014.

Revision received: 27 January 2015.

Accepted for publication: 31 January 2015.

This work is licensed under a Creative Commons Attribution 3.0 License (by-nc 3.0).

(C) Copyright S. Marozzi et al., 2015

Licensee PAGEPress, Italy

Italian Journal of Food Safety 2015; 4:4567

doi:10.4081/ijfs.2015.4567

un arco temporale ravvicinato di tre ceppi di $L$. monocytogenes nel medesimo presidio ospedaliero ha comportato l'esecuzione di un'indagine epidemiologica, ambientale e sugli alimenti e la richiesta di analisi di laboratorio tra cui l'identificazione sierologia e tipizzazione molecolare dei ceppi, al fine di identificare 0 escludere eventuali correlazioni tra i casi. Sono state quindi eseguite la determinazione degli antigeni somatici e flagellari con kit Listeria Antisera SEIKEN (DENKA SEIKEN CO. Ltd, Tokyo, Giappone) e la tipizzazione molecolare mediante pulsed field gel electrophoresis (PFGE) secondo il protocollo standardizzato PulseNet (CDC, 2009). Per la PFGE è stato impiegato l'enzima di restrizione (AscI) ed uno standard riferibile a Salmonella Braenderup (H9812). Dopo la digestione, i ceppi sono stati sottoposti a corsa elettroforetica con il sistema Chef Mapper XA (BioRad Inc., Berkeley, CA, Stati Uniti) a $6 \mathrm{~V} / \mathrm{cm}$ con un switch time da 4 a $40 \mathrm{~s}$ per $21 \mathrm{~h}$. Il gel ottenuto è stato, quindi, colorato con il GelRed (Biotium, Hayward, CA, Stati Uniti). I profili sono stati analizzati mediante software dedicato (Fingerprinting $\mathrm{II}^{\circledR} \mathrm{BioRad}$ ). La valutazione della similarità tra i diversi profili di macrorestrizione è stata effettuata utilizzando il coefficiente di Dice con una ottimizzazione e tolleranza pari all'1\%. La clusterizzazione e la costruzione del dendrogramma sono state effettuate utilizzando metodo unweighted pair group method with arithmetic mean.

Contemporaneamente alle indagini di laboratorio, sono stati condotti dall'ASL Roma/B alla quale afferisce la struttura sanitaria d'invio dei ceppi, gli accertamenti epidemiologici, ambientali ed alimentari. In seguito agli stessi è emerso che la donna risultata positiva per Listeria, aveva consumato qualche settimana prima del parto, avvenuto pre-termine (34 
esima settimana e $3 \mathrm{gg}$ ), alcuni alimenti a rischio per la presenza di $L$. monocytogenes ovvero: stracchino, gorgonzola e salmone affumicato, tutti acquistati in un esercizio di vendita al dettaglio della grande distribuzione. A proposito degli alimenti, la donna riferiva la marca dello stracchino ma non quella del gorgonzola e del salmone affumicato, tuttavia per quanto concerne quest'ultimo ricordava chiaramente la tipologia (dimensioni, colore delle scritte e delle stampe) della confezione.

In seguito alle dichiarazioni della madre è stato prelevato dai Servizi Veterinari della Roma/B, nell'esercizio in questione, un campione reperto di salmone affumicato che, per packaging, presentava le caratteristiche descritte dalla donna. Sullo stesso sono state condotte le seguenti analisi di laboratorio: determinazione dell' $\mathrm{a}_{\mathrm{w}}$ mediante metodo di riferimento ISO 21807:2004 (IS0, 2004), valutazione del pH secondo il metodo MFHPB 03:2012 (Health Canada, 2012), esame colturale qualitativo e quantitativo per la ricerca di $L$. monocytogenes come da metodo UNI EN ISO 11290:2005 parte 1 e 2 (UNI, 2005). Per l'identificazione sierologica sui ceppi isolati è stato infine eseguito un test di sieroagglutinazione con kit Listeria Antisera SEIKEN (DENKA SEIKEN CO. Ltd) ed una tipizzazione molecolare mediante PFGE secondo il protocollo standardizzato PulseNet (CDC, 2009), operando come precedentemente descritto.

\section{Risultati}

La sieroagglutinazione ha consentito di attribuire tutti i tre ceppi umani isolati al sierotipo 4b. La tipizzazione molecolare con PFGE ha invece evidenziato l'appartenenza a due cluster differenti ovvero cluster B per gli isolati da madre e figlio e cluster A per quello ottenuto dal secondo neonato. Per quanto concerne infine i risultati degli esami analitici condotti sul campione reperto di salmone affumicato, in Tabella 1 vengono riportati i valori di $\mathrm{a}_{\mathrm{w}}, \mathrm{pH} \mathrm{e}$ gli esiti degli esami colturali per la ricerca di $L$. monocytogenes. In particolare sono risultate contaminate da $L$. monocytogenes 4 unità cam- pionarie su 5 testate all'esame qualitativo ed 1 unità campionaria su 5 all'esame quantitativo con valori di $4,5 \times 10 \mathrm{ufc} / \mathrm{g}$.

La sierotipizzazione ha identificato tutti i ceppi isolati dall'alimento come appartenenti al sierotipo 1/2A, mentre la tipizzazione molecolare ha evidenziato un ulteriore cluster diverso da quelli di appartenenza degli isolati umani.

\section{Discussione}

Come evidenziato dalla presenza di due differenti fingerprinting ottenuti con PFGE, non è stata rilevata una correlazione epidemiologica tra i due casi di listeriosi neonatale pur avendo la sieroagglutinazione rilevato l'appartenenza dei tre ceppi al medesimo sierotipo 4b. Alcuni autori hanno evidenziato una correlazione tra sierotipi e quadro clinico della malattia, in particolare il sierotipo 4b è stato associato alle listeriosi gravidiche. L'incidenza del sierotipo 4b nei casi di listeriosi varia dal 50 al 70\% e tende a essere più elevata negli episodi perinatali (Katarihou, 2002). Per quanto riguarda invece i ceppi isolati dal campione reperto di salmone affumicato, l'appartenenza al sierotipo 1/2a ha escluso ogni correlazione tra i casi umani e il consumo dell'alimento.

Il tasso di mortalità elevato causato dalla listeriosi ha indotto i governi e le agenzie per la sicurezza alimentare di numerose nazioni nel mondo a prendere misure per ridurre il rischio correlato alla presenza di $L$. monocytogenes negli alimenti. Nell'Unione Europea il Regolamento 2073/2005 (Commissione Europea, 2005) e successive modifiche e integrazioni ha ampliato il campo di tolleranza per i RTE in caso di prodotti immessi sul mercato sino a valori di 100 unità formanti colonia (ufc)/g. Tuttavia, questo limite viene applicato solo se il produttore è in grado di dimostrare, con soddisfazione dell'Autorità Competente, che l'alimento non supera tale soglia al termine della shelf-life. Da un punto di vista legale il campione reperto di salmone affumicato era conforme alle disposizioni vigenti in materia, così come avvalorato dai Servizi Veterinari competenti per territorio, che hanno visionato la documentazione prodotta dalla ditta. Tuttavia sono doverose alcune osservazioni. In primo luogo in base ai risultati delle indagini condotte non è stato possibile rintracciare il lotto di produzione del salmone affumicato consumato dalla madre. Un'altra considerazione, a prescindere dalla mancata correlazione epidemiologica tra alimento e casi umani, scaturisce dall'esito della analisi quantitativa (ufc/g). La relazione dose-risposta per la listeriosi è sconosciuta, tuttavia è accertato che questa è dipendente dallo stato immunitario dell'ospite e dalla virulenza del ceppo. Secondo la valutazione della Food and Agriculture Organization (FAO) e del WHO (FAO/WHO, 2004), casi gravi di malattia possono manifestarsi nell'1\% della popolazione sana con valori di $4 \times 10^{11}$ cellule, valore che diminuisce a $10^{10}$ cellule per le categorie sensibili (per es. immunodepressi). La valutazione del rischio legato alla presenza di 4,5×10 ufc/g di $L$. monocytogenes in un alimento considerato favorevole al suo sviluppo, quale il salmone affumicato (EFSA, 2014), deve tener conto della porzione consumata e delle variabili associate alla conservazione casalinga dell'alimento e alla condizione individuale di chi lo consuma. In generale, come riportato dalla FAO/WHO (2004), i livelli di L. monocytogenes negli alimenti implicati in corso di tossinfezione superano $10^{3} \mathrm{ufc} / \mathrm{g}$, sebbene in alcuni episodi siano stati riscontrati livelli di contaminazione sostanzialmente inferiori. Inoltre, il rischio calcolato di contrarre listeriosi assumendo il consumo di una porzione di $36 \mathrm{~g}$ di alimento con un livello di contaminazione pari a $100 \mathrm{ufc} / \mathrm{g}$ è di $1,85 \times 10^{-9}$ casi per porzione. Tale rischio aumenta in caso di salmoni affumicati a freddo $\left(5,3 \times 10^{-8}\right.$ casi per porzione).

\section{Conclusioni}

La corretta identificazione dell'alimento veicolo di tossinfezione, avrebbe dovuto implicare il campionamento dei due formaggi che la donna riferiva di aver consumato (stracchino e gorgonzola). Purtroppo come spesso accade in

Tabella 1. Risultati delle analisi condotte sulle cinque unità del campione di salmone affumicato.

\begin{tabular}{lcccc} 
Unità campionaria & $\mathrm{a}_{\mathrm{w}}$ & $\mathrm{pH}$ & $\begin{array}{c}\text { Esame colturale per la ricerca di Listeria monocyotgenes } \\
\text { Qualitativo }\end{array}$ & Presente in $25 \mathrm{~g}$ \\
1 & 0.972 & 5.9 & $<10 \mathrm{ufc} / \mathrm{g}$ & Presente in $25 \mathrm{~g}$ \\
\hline 3 & 0.966 & 6.0 & $<10 \mathrm{ufc} / \mathrm{g}$ & Presente in $25 \mathrm{~g}$ \\
4 & 0.979 & 6.0 & $<10 \mathrm{ufc} / \mathrm{g}$ & Presente in $25 \mathrm{~g}$ \\
\hline 5 & 0.967 & 6.0 & $4,5 \times 10^{1} \mathrm{ufc} / \mathrm{g}$ & Assente in $25 \mathrm{~g}$ \\
\hline
\end{tabular}


corso di tossinfezione alimentare, gli alimenti che la donna riferiva di aver consumato erano irreperibili nell'esercizio dove la stessa li aveva acquistati. Come è noto i formaggi figurano tra gli alimenti a maggior frequenza di positività per L. monocytogenes (EFSA, 2104) e sono, di conseguenza, chiamati in causa in numerosi episodi epidemici e sporadici di tossinfezione alimentare. Non è da escludere a priori, pertanto, che questi fossero coinvolti nella trasmissione del microrganismo. Infine, è degna di nota la limitazione nell'investigazione del caso sporadico riferibile al secondo neonato causata dalla presenza di un'indagine giudiziaria in corso. Questa circostanza ha reso gli atti investigativi dei nuclei antisofisticazione e sanità non consultabili.

\section{Bibliografia}

Allerberger F, Wagner M, 2010. Listeriosis: a resurgent foodborne infection. Clin Microbiol Infec 16:16-23.

ANSES, 2011. Data sheet on foodborne biological hazard. Listeria monocytogenes.
Disponibile al sito: http://www.afssa.fr/ Documents/MIC-Fi-ListeriaEN.pdf

CDC, 2009. Standardized laboratory protocol for molecular subtyping of Listeria monocytogenes by pulsed field gel electrophoresis (PFGE). Disponibile al sito: http://www.cdc.gov/pulsenet/protocols/ pulsenet_listeria_protocol\%20.pdf

Commissione Europea, 2005. Regolamento della Commissione del 15 novembre 2005 sui criteri microbiologici applicabili ai prodotti alimentari, 2073/2005/CE. In: Gazzetta Ufficiale, L 338/1, 22.12.2005.

EFSA, 2014. The European Union summary report on trends and sources of zoonoses, zoonotic agents and food-borne outbreaks in 2012. EFSA J 12:3547.

FAO/WHO, 2004. Risk assessment of Listeria monocytogenes in ready-to-eat foods: interpretative summary. Disponibile al sito: ftp://ftp.fao.org/es/esn/jemra/mra4 _en.pdf

ISO, 2004. Microbiology of food and animal feeding stuffs. Determination of water activity. Norma ISO 21807:2004. Organizzazione Internazionale per la Standardizzazione, Ginevra, Svizzera.

Kathariou S, 2002. Listeria monocytogenes vir- ulence and pathogenicity, a food safety perspective. J Food Protect 65:1811-29.

Mead PS, Slutsker L, Dietz V, McCaig LF, Bresee JS, Shapiro C, Griffin PM, Tauxe RV, 1999. Food-related illness and death in the United States. Emerg Infect Dis 5:60725.

Health Canada, 2012. Determination of the $\mathrm{pH}$ of foods including foods in hermetically sealed containers. Method MFHPB03:2012. Health Canada, Ottawa, Canada.

Lamont RF, Sobel J, Mazaki-Tovi S, Kusanovic JP, Vaisbuch E, Kim SK, Uldbjerg N, Romero R, 2011. Listeriosis in human pregnancy: a systematic review. J Perinat Med 39:227-36.

UNI, 2005. Norma UNI EN ISO 11290:2005. Microbiologia di alimenti e mangimi per animali - Metodo orizzontale per la ricerca e la conta di Listeria monocytogenes. Parte 1: metodo per la ricerca. Parte 2: metodo per la conta. Ente Italiano di Normazione, Milano, Italy.

WHO, 2008. Foodborne disease outbreaks. Guidelines for inverstigation and control. Disponibile al sito: www.who.int/foodsafety/publications/foodborne_disease/outbreak_guidelines.pdf 